\title{
Protocol
}

\section{In Vivo Imaging of Cerebral Hemodynamics Using High-Frequency Micro-Ultrasound}

\author{
John Sun, ${ }^{1,3}$ Liis Lindvere, ${ }^{1,2}$ Martijn E. van Raaij,, ${ }^{1}$ Adrienne Dorr, ${ }^{1}$ \\ Bojana Stefanovic, ${ }^{1,2}$ and F. Stuart Foster ${ }^{1,2}$ \\ ${ }^{1}$ Imaging Research, Sunnybrook Health Sciences Centre, Toronto, Ontario M4N 3M5, Canada \\ ${ }^{2}$ Department of Medical Biophysics, University of Toronto, Toronto, Ontario M5G 2M9, Canada
}

\section{INTRODUCTION}

Assessment of cerebral vascular response is important in neuroscience research. Some imaging modalities that are commonly used to detect flow and/or vessel diameter changes in the brain include magnetic resonance imaging, positron emission tomography, and optical intrinsic signal imaging. Ultrasound has not typically been used to assess neurovascular response but recent advances in the technology have led to the development of micro-ultrasound systems with significant potential for this application. The state of the art in high frequency $(15-50 \mathrm{MHz})$ micro-ultrasound is based on linear arrays specifically designed for small animal imaging. These systems can achieve axial resolution ranging from 30 to $200 \mu \mathrm{m}$. They are capable of quantifying brain hemodynamics in terms of red blood cell (RBC) velocity, flow, and vascular density in real time, up to $35 \mathrm{~mm}$ below the cortical surface, and can achieve temporal resolution of up to 1000 frames per second. This protocol describes imaging of the rat brain using various ultrasound imaging modes (power Doppler, color Doppler, pulsed-wave Doppler, and nonlinear contrast-enhanced imaging) to assess the state of cerebral microcirculation.

\section{RELATED INFORMATION}

The frequency of ultrasound scattered by RBCs and detected by an ultrasound imaging system is proportional to the velocity of the RBCs relative to the ultrasound source and detector (transducer). This effect, known as the Doppler effect, creates an opportunity to probe aspects of microcirculatory flow in the brain with very high spatial and temporal resolution. Doppler ultrasound techniques are particularly well suited to the cerebral cortex because many penetrating vessels are largely perpendicular to the surface, aligning the axis of observation with the flow direction; this maximizes the Doppler frequency shift.

\section{Power Doppler Imaging}

It has been shown previously that the power in the Doppler signal is proportional to the density of RBCs in tissues (Nelson and Pretorius 1988). Power Doppler, therefore, assuming constant hematocrit, reflects relative blood volume in the region being imaged. Power Doppler maps can be two- or threedimensional (Fig. 1). In Figure 1, the power Doppler signal is displayed on an orange-to-yellow color scale with dark orange representing low Doppler power and low relative blood volume; yellow represents high Doppler power and high relative blood volume. Therefore, this technique can provide a morphological image of the microvasculature in the imaging plane or volume of interest. The ultrasound system software also affords calculation of percent vascularity (PV), a relative measure of

${ }^{3}$ Corresponding author (jsun@sri.utoronto.ca). 

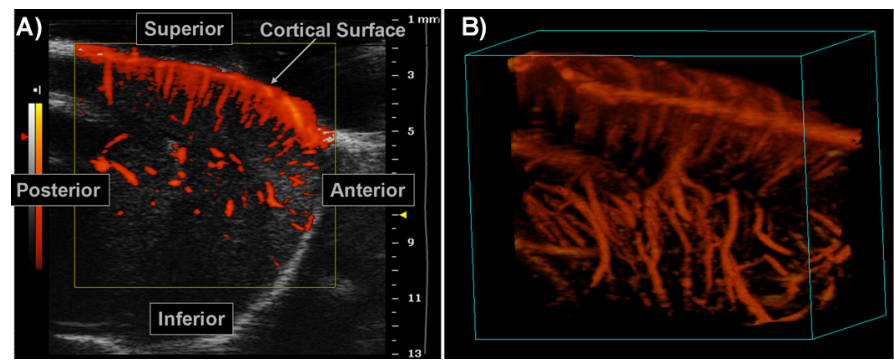

FIGURE 1. (A) Two- and (B) three-dimensional-rendered power Doppler images of the rat cerebrovasculature.

vascular density in the imaging plane or volume of interest. It is obtained by calculating the normalized power Doppler signal within a user-defined region of interest. Although power Doppler provides a simple rapid mean of estimating blood volume, it is limited in that slow flow (e.g., such as that in vessels smaller than $\sim 50 \mu \mathrm{m}$ in diameter) might not meet the power Doppler detection threshold. In addition, it is prone to motion artifacts that are not related to RBC motion.

\section{Color Doppler Imaging}

Color Doppler imaging detects mean RBC velocity and is a directional measurement (Burns 1987). It distinguishes flow toward (red/yellow color scale) and away from (blue/turquoise color scale) the transducer. In imaging the brain cortex, this provides a useful method for separating penetrating arterioles from venules that are roughly perpendicular to the cortical surface. In a color flow image of the cortex (Fig. 2), the arterial component (blue) shows average RBC velocities in the range of 0-5 mm/sec. Similar velocities are observed in the opposite direction for venous vessels (red). Although the RBC speed in arterioles is on average higher than those in the venules of similar caliber, considerable overlap in the two ranges has been reported and the present values agree well with the previously reported speeds of $0.1-6 \mathrm{~mm} / \mathrm{sec}$ in the penetrating arterioles and of $1.9-6 \mathrm{~mm} / \mathrm{sec}$ in the pial venules (Rosenblum 1975; Thomale et al. 2001; Nishimura et al. 2007). A region of high velocity flow is visible centrally and is identified by its chaotic color patterns resulting from a sampling artifact called aliasing. It is important to note that color Doppler is not quantitative. It serves only to provide a pictorial overview of the relative velocities. If accurate and reliable quantification of RBC velocity is needed, pulsed Doppler imaging should be used.

\section{Pulsed-Wave Doppler Imaging}

Pulsed-wave Doppler imaging displays flow velocity vs. time waveform at one point in space by measuring the Doppler shift from the ultrasound backscatter of RBCs (Burns 1987). This provides a more detailed and accurate RBC velocity in a vessel of interest relative to color Doppler imaging. Figure 3 shows an example of a pulsed Doppler measured near the cortical surface in one of the arterial feeding vessels. At this location, peak RBC velocities of over $100 \mathrm{~mm} / \mathrm{sec}$ are observed. The temporal shape

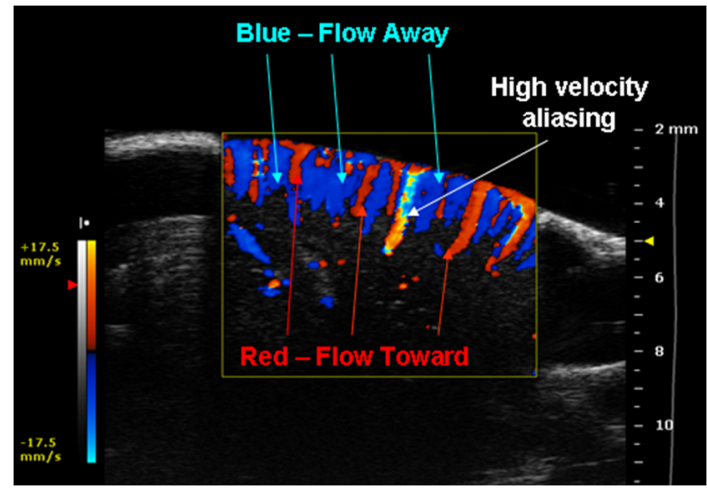

FIGURE 2. Color Doppler image overlayed on a B-mode image of rat cortex. Blue and red signals represent flow away from and toward the transducer, respectively. When very high velocity is present, aliasing occurs. (For color figure, see doi: 10.1101/pdb.prot5495 online at www.cshprotocols.org.) 
of the Doppler waveform shows significant cardiac pulsatility. Furthermore, if the vessel cross-sectional area can be determined, then flow rate (e.g., in milliliter/minutes) can also be calculated.

\section{Contrast-Enhanced Imaging}

Contrast-enhanced imaging requires intravenous infusion of ultrasound contrast agents. The protocol described here uses MicroMarker: lipid-shelled microspheres $\sim 2.5 \mu \mathrm{m}$ in diameter containing perfluoropentane gas. These microspheres generate far greater ultrasound backscatter than RBCs or surrounding tissue. Therefore, after infusing these microspheres, there is a significant enhancement in signal intensity from blood vessels. By plotting the signal intensity as a function of time, blood flow and volume can be assessed (Wei et al. 1998; Hudson et al. 2009). The protocol described here uses "nonlinear" contrast imaging, which takes advantage of the fact that ultrasound contrast agents reflect ultrasound differently than biological tissue. Nonlinear contrast detection suppresses the tissue signal and isolates signal from the contrast microspheres, yielding enhanced sensitivity to blood flow (Needles et al. 2009). In this protocol, contrast agent is infused intravenously slowly and continuously using a syringe infusion pump. Once the contrast agent concentration in the systemic circulation reaches a steady state, a high-intensity ultrasound pulse is applied that instantaneously disrupts all contrast agent within the imaging plane. Because the disruption is applied only in one specific two-dimensional slice $(\sim 200 \mu \mathrm{m}$ thick), there is a rapid ( 2 sec) contrast recovery as microspheres return to this slice. This process is illustrated schematically in Figure 4. The initial slope of the replenishment is proportional to flow, whereas the plateau is proportional to the vascular volume. An example of a disruption/replenishment (Fig. 5) shows the quantitative inflow kinetics for two regions. The replenishment reveals varying levels of plateau values: Region 1 has a higher plateau value, suggesting it has a larger vascular volume among the chosen regions.

An alternative means of imaging intact cortex in situ with high temporospatial resolution is described in Two-Photon Fluorescence Microscopy of Cerebral Hemodynamics (Lindvere et al. 2010).

\section{MATERIALS}

CAUTIONS AND RECIPES: Please see Appendices for appropriate handling of materials marked with $<!>$, and recipes for reagents marked with $<\mathbf{R}>$.

For additional reagents and equipment required for animal preparation and physiological monitoring, see TwoPhoton Fluorescence Microscopy of Cerebral Hemodynamics (Lindvere et al. 2010).

\section{Reagents}

$<$ !>Rats, Sprague-Dawley, male, 120-150 g (Charles River Laboratories Inc., St-Constant, Quebec, Canada)

Saline (for contrast-enhanced imaging only)

Ultrasound contrast agent (for contrast-enhanced imaging only) (MicroMarker; VisualSonics Inc.)

Ultrasound gel

\section{Equipment}

Coverslip, Rexolite, $12 \times 6 \times 0.1 \mathrm{~mm}$ (C-Lec)

Imaging platform (Integrated Rail System; VisualSonics Inc.)

Micro-ultrasound system, high-frequency (Vevo2100; VisualSonics Inc.)

Needles, 21-gauge (for contrast-enhanced imaging only)

Syringe infusion pump (NE-1000; New Era Pump Systems) (for contrast-enhanced

imaging only)

Syringes, 1-mL (for contrast-enhanced imaging only)

Timer

Ultrasound transducers, 20 and $40 \mathrm{MHz}$ (MS250 and MS550S, respectively; VisualSonics Inc.) 


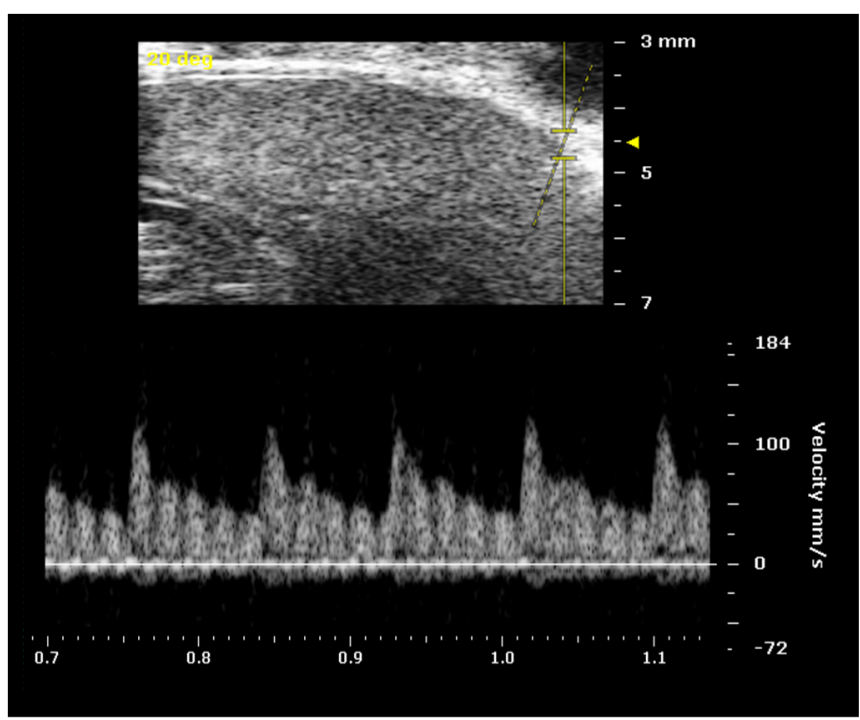

FIGURE 3. Pulsed-wave Doppler image (top) showing flow velocity vs. time profile (bottom) of the cerebral surface vessel.

\section{METHOD}

Separate procedures are described for power and color Doppler imaging (Steps 7-13), pulsed-wave Doppler imaging (Steps 14-21), and contrast-enhanced imaging (Steps 22-25). These procedures presume the investigator has basic knowledge of the operation of the VisualSonics Vevo2100 ultrasound system (see http://www2.healthsci.tufts.edu/ saif/Vevo2100/PN\%2011853\%20Vevo\%202100\%200perator\%20Manual\%20Rev\%201.1.pdf).

\section{Surgical Procedure}

Procedures for the humane treatment of animals must be observed at all times. Consult your local animal facility for guidelines. The experimental procedures performed in this study have been reviewed and approved by the Animal Care Committee at Sunnybrook Research Institute, Toronto.

1. Perform tracheotomy, femoral artery, femoral vein and tail vein cannulation, craniotomy, and physiological monitoring and maintenance as described in Two-Photon Fluorescence Microscopy of Cerebral Hemodynamics (Lindvere et al. 2010).

2. Following craniotomy, apply a few drops of body-temperature $1 \%(w / v)$ agarose to the exposed brain.

3. Place a sterile Rexolite cover over the window. Glue the perimeter of the coverslip to the skull using cyanoacrylate glue. Allow the adhesive to dry completely.

Because the glue can leak under the coverslip, let it sit in a weigh boat for a few minutes before application so that it becomes more viscous.

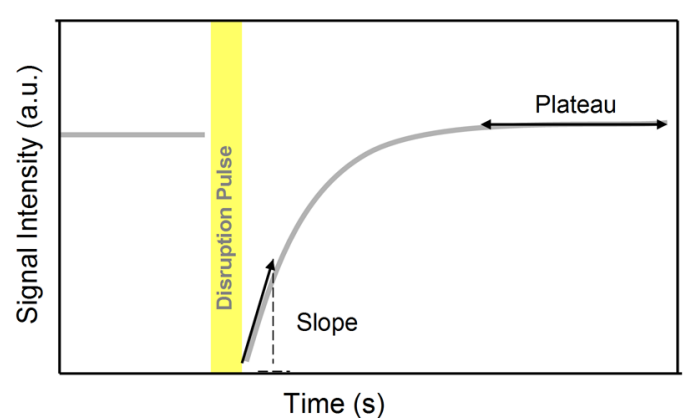

FIGURE 4. Schematic of a contrast agent disruption/replenishment curve. Slope and plateau are relative measures of flow and vascular volume, respectively. 
A)

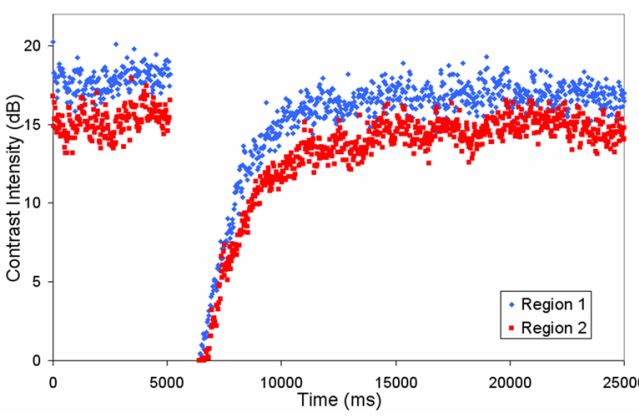

B)

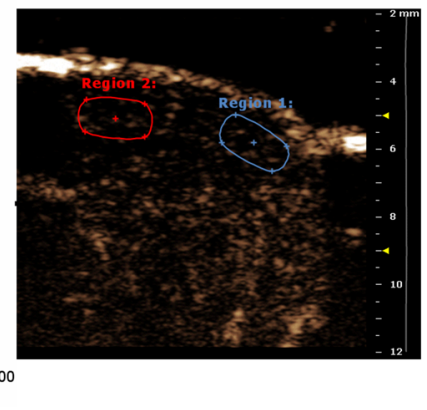

FIGURE 5. (A) Typical disruption/replenishment curves. (B) Two different regions of interest are displayed, revealing different replenishment curve plateaus. (For color figure, see doi: 10.1101/pdb.prot5495 online at www.cshprotocols.org.)

4. Perform blood gas analysis. Take any corrective actions as needed.

5. Switch animal to $\alpha$-chloralose anesthetic for imaging:

i. Administer an $80-\mathrm{mg} / \mathrm{kg}$ bolus at room temperature over the course of a few minutes while simultaneously decreasing isoflurane. Monitor the heart rate and $\mathrm{O}_{2}$ saturation closely during the transition.

The bolus allows for $15 \mathrm{~min}$ of an appropriate level of anesthesia (Flecknell 1996).

ii. Infuse $\alpha$-chloralose continuously through the femoral vein at a rate of $27 \mathrm{mg} / \mathrm{kg} / \mathrm{h}$ for the remainder of the experiment.

6. Use the stereotaxic frame to position the ultrasound probe at the site of interest (Fig. 6).

\section{Power and Color Doppler Imaging}

Optimize settings for power and color Doppler imaging in the rat brain as described below. Although other parameters (e.g., Doppler gain, transmit frequency, sensitivity, persistence, priority, and transmit power) can also affect Doppler imaging, the system's default settings for these are usually adequate for imaging the rat brain. Ensure the settings used remain consistent for all animals, because changes in any parameters can directly alter estimated values.

7. Initiate the $40-\mathrm{MHz}$ ultrasound transducer.

8. Set the pulse repetition frequency (PRF):

If detection of slow velocity $(<15 \mathrm{~mm} / \mathrm{sec})$ is required

i. Set the PRF to $1.0 \mathrm{kHz}$.

ii. Gradually increase the PRF until an acceptable signal-to-noise ratio is achieved.

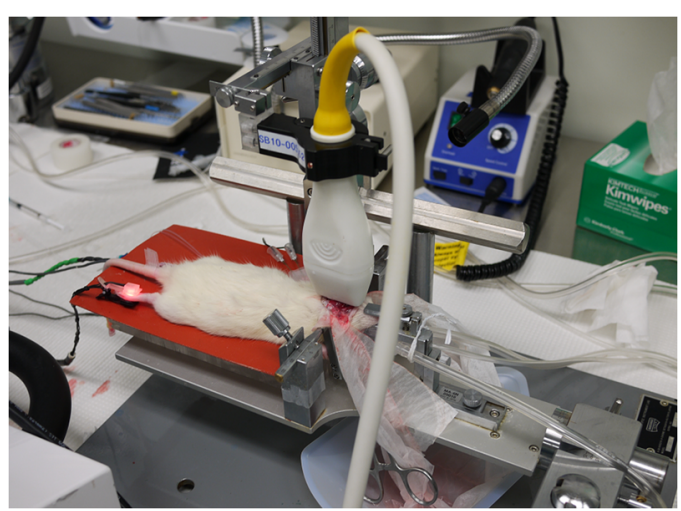

FIGURE 6. Photograph of the experimental system. At this point in the experiment, craniotomy has been performed and the ultrasound transducer is placed stereotaxically above the cortical surface. 


\section{If preferential detection of fast velocity $(>50 \mathrm{~mm} / \mathrm{sec})$ vessels is required}

iii. Perform Steps 8.i.-ii.

iv. Continue to increase the PRF until the Doppler signal in the slower flow vessels disappears. The typical PRFs required for slow and fast velocities are 1.5 and $5.0 \mathrm{kHz}$, respectively.

9. Set the wall filter.

Power Doppler is very motion-sensitive. Minor vibrations caused by nearby mechanical devices or closing and opening of nearby doors can introduce noise. If the source of the unwanted motion cannot be eliminated, a wall filter can be implemented to minimize the resultant noise at the expense of the system's sensitivity to detect slow velocities.

10. Set the sample volume (SV) gate:

To maximize signal resolution

i. Set the SV gate to low.

To maximize flow detection sensitivity

ii. Set the SV gate to high.

11. Adjust the focus depth to the depth of interest.

12. To maximize temporal resolution, limit the field of view to the region of interest. The Doppler imaging frame rate is greatly affected by the field-of-view.

13. Trace the region of interest. Perform a power signal histogram analysis to generate a PV value. In three-dimensional power Doppler imaging, PV is generated once a volume of interest is defined.

\section{Pulsed-Wave Doppler Imaging}

14. Initiate the $40-\mathrm{MHz}$ ultrasound transducer.

15. Locate the vessel of interest (e.g., via color Doppler imaging).

16. Place the pulsed-wave Doppler overlay on the vessel of interest. Activate pulsed-wave Doppler imaging.

17. Align the angle correction parallel to the vessel of interest. Minimize this angle by adjusting the transducer orientation if possible. If the angle is $>75^{\circ}$, activate the angle steering to decrease this angle.

Smaller angle corrections yield more accurate velocity estimates.

18. Adjust the SV size according to vessel size.

19. Adjust the PRF to an appropriate value for the RBC velocity of interest.

20. Increase the Doppler gain if the signal is faint.

21. Use the software measurement tool to quantify velocity values.

\section{Contrast-Enhanced Imaging}

22. Prepare the contrast agent for infusion:

The MicroMarker contrast agent comes in a dry-freeze powder format and must be reconstituted with saline before injection. There are $2 \times 10^{9}$ microbubbles in each vial of MicroMarker.

i. Using a 1-mL syringe and a 21 -gauge needle, inject $1000 \mu \mathrm{L}$ of saline into the vial.

ii. Remove the syringe. Leave the needle in the vial for $\sim 2 \mathrm{sec}$ to relieve the added pressure. Ultrasound microbubbles can be destroyed under high-pressure environments.

iii. Gently shake the vial for $\sim 1$ min to mix the contents.

iv. Using a new 1-mL syringe and a 21-gauge needle, withdraw $\sim 300 \mu \mathrm{L}$ of this mixture. 
This is the contrast infusion syringe.

v. Set up the infusion syringe on the infusion pump. Attach to the tail vein catheter.

23. Set up the ultrasound, as follows:

Other parameters such as dynamic range, sensitivity, SV gate, persistence, and transmit frequency can also affect contrast imaging, but the system's default settings should be adequate for contrast imaging in the rat brain. Ensure all settings used remain consistent for all animals, because changes in any parameters can directly alter signal intensity values.

i. Initialize the 20-MHz ultrasound transducer.

ii. Set the transmit beam width to "Wide."

iii. Shift all time-gain-compensation (TGC) sliders to the exact middle position.

iv. Set up an "imaging sequence" loop that contains a contrast disruption pulse.

v. Adjust the gain to the desired level.

Increasing gain can increase signal at the expense of increasing tissue noise.

vi. Set two focal zones. Adjust to the depth of interest.

vii. Set the line density to "High."

viii. Set the transmit power to $4 \%$.

24. Perform a time-to-reach-stability test:

This test estimates the time required (from the start of infusion) for the contrast agent concentration to reach a steady state in the rat circulation. Once steady state is reached, a disruption pulse can be applied to acquire a replenishment intensity curve.

i. Simultaneously, start the infusion pump, begin imaging, and start a timer.

Signal intensity should increase gradually as contrast agent begins to flow into the brain.

ii. To determine when signal intensity reaches a plateau value, use the contrast region trace and plot a signal intensity curve.

iii. Note the time required to reach a stable intensity.

iv. Continue to monitor the signal intensity until it begins to decrease, noting this time as well.

In our experience, the time required to reach steady state is 3-5 min, and steady state lasts for 1-2 min.

25. Perform disruption/replenishment acquisition:

Disruption/replenishment acquisition should be performed following the establishment of plateau signal intensity. If addition of a stimulus (e.g., $\mathrm{CO}_{2}$ inhalation, electrical stimulation to the forepaw) is desired to assess vascular reactivity, time the introduction of the stimulus such that the cerebral hemodynamics are in steady state during the disruption/replenishment process.

\section{i. Begin contrast infusion.}

ii. Thirty seconds following plateau establishment, image the disruption/replenishment.

This can be repeated if replicate measurements or estimates of cerebral state under varying conditions are required. However, ensure that all image sequence acquisitions are complete before the signal starts to decline.

iii. To perform reperfusion analysis, use the contrast region trace tool to trace the regions of interest and plot contrast intensity curves.

Flow dynamics can also be assessed with a continuous infusion without the disruption/replenishment event. Once plateau intensity is established, a stimulus can be introduced and a response observed by an immediate change in plateau intensity, signifying a change in relative blood volume. However, because no replenishment is observed in this method, change in flow cannot be measured directly.

\section{DISCUSSION}

Micro-ultrasound is a novel technique for assessing cerebrovascular hemodynamics in the rodent. Like other techniques such as two-photon fluorescence microscopy, this imaging technique is only 

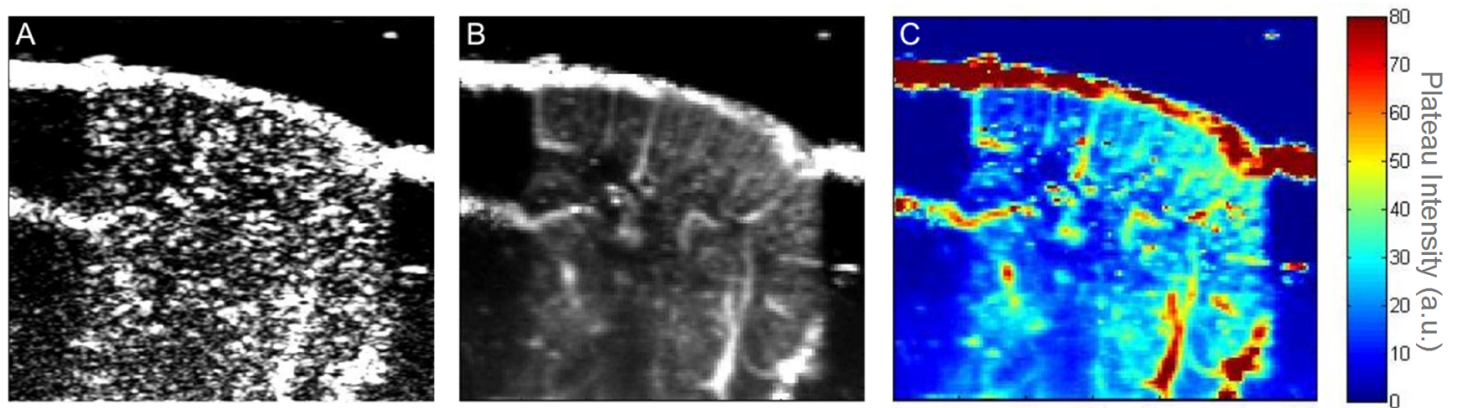

FIGURE 7. Voxel-by-voxel image analysis of nonlinear contrast disruption/replenishment image sequences can yield vessel-specific information. (A) A single frame from a 1000-frame sequence. (B) Maximum intensity projection showing the trajectories of the bubbles, i.e., the location of the blood vessels. (C) A parametric map of the parameter $A$ in the equation $I=A\left(1-\mathrm{e}^{-\beta t}\right)$ (Wei et al. 1998) as fitted to the replenishment curve, indicating relative vascular density in the imaging plane. (For color figure, see doi: 10.1101/pdb.prot5495 online at www.cshprotocols.org.)

possible with the surgical preparation of a flawless craniotomy. Whereas two-photon fluorescence microscopy permits very high resolution imaging of vascular structure and response over a shallow depth, micro-ultrasound provides 30-200- $\mathrm{mm}$ resolution over several centimeters of brain tissue. In addition, the real-time nature of ultrasound opens up the possibility of observing vascular response to various stimuli with a very high temporal resolution.

This protocol describes the measurement of hemodynamic parameters using Doppler and nonlinear contrast techniques. Power Doppler reports primarily on blood volume and provides a topological map of the vascular tree down to $\sim 100 \mu \mathrm{m}$. However, power Doppler does not have sufficient sensitivity to detect flow in the capillary microcirculation and is therefore of limited use in the investigation of microvascular flow. Color Doppler imaging adds additional functionality in the form of directional information on blood flow in the penetrating supply and draining vessels of the cortex. These vessels tend to run perpendicular to the cortical surface and are therefore ideal candidates for Doppler investigation because the Doppler angle is close to zero and the recorded velocities are inherently accurate. More detailed analysis of the time variation of blood velocity is possible using pulsed-wave Doppler, in which the operator selects a specific location in the image plane for this analysis. Ultrasound contrast agents fill a gap left by the Doppler techniques by allowing investigation of hemodynamics at all levels of the circulation. Furthermore, the ability to selectively disrupt microbubbles with transient high powered ultrasound pulses means that the inflow kinetics to selected regions, usually a two-dimensional image slice, can be probed for local perfusion and blood volume measurements.

High-frequency micro-ultrasound imaging of the rodent cerebrum produces a rich data set. Even though tissue contrast in the brain is limited, contrast-enhanced image sequences can be processed to yield images of blood vessel topography (Fig. 7). Figure 7B shows microbubble trajectories detailing the architecture of the microcirculation. Voxel-by-voxel analysis of disruption/replenishment kinetics can produce parametric maps of blood flow and volume (Fig. 7C). Together with the Doppler images discussed above, contrast-enhanced high-frequency micro-ultrasound promises to be a valuable addition to the study of cerebral hemodynamics.

\section{ACKNOWLEDGMENTS}

We thank the Terry Fox Foundation, Canadian Institutes for Health Research, the Ontario Research Fund, and VisualSonics for support of this work. F.S.F. discloses a financial interest in VisualSonics.

\section{REFERENCES}

Burns PN. 1987. The physical principles of Doppler and spectral analysis. J Clin Ultrasound 15: 567-590.

Flecknell P. 1996. Laboratory animal anaesthesia: A practical introduction for research workers and technicians, 2nd ed. Academic Press, London, UK.
Hudson JM, Karshafian R, Burns PN. 2009. Quantification of flow using ultrasound and microbubbles: A disruption replenishment model based on physical principles. Ultrasound Med Biol 35: 2007-2020.

Lindvere L, Dorr A, Stefanovic B. 2010. Two-photon fluorescence 
microscopy of cerebral hemodynamics. Cold Spring Harb Protoc (this issue). doi: $10.1101 /$ pdb.prot5494.

Needles A, Mehi J, Arditi M, Gaud E, Frinking P, Rognin N, Hirson D, Foster S. 2009. Nonlinear contrast agent imaging with a linear array based micro-ultrasound. pp. 362-363. 2009 IEEE International Ultrasonics Symposium Proceedings, Rome, Italy. Nelson TR, Pretorius DH. 1988. The Doppler signal: Where does it come from and what does it mean? A/R Am / Roentgenol 151: 439-447.

Nishimura N, Schaffer CB, Friedman B, Lyden PD, Kleinfeld D. Penetrating arterioles are a bottleneck in the perfusion of neocortex. Proc Natl Acad Sci 104: 365-370.
Rosenblum WI. 1975. Effect of pial arteriolar constriction on red cell velocity in pial venules and on venular diameter. Microvasc Res 9: $38-42$.

Thomale UW, Schaser KD, Unterberg AW, Stover JF. 2001. Visualization of rat pial microcirculation using the novel orthogonal polarized spectral (OPS) imaging after brain injury. I Neurosci Methods 108: 85-90.

Wei K, Jayaweera AR, Firoozan S, Linka A, Skyba DM, Kaul S. 1998. Quantification of myocardial blood flow with ultrasound-induced destruction of microbubbles administered as a constant venous infusion. Circulation 97: 473-483. 


\section{In Vivo Imaging of Cerebral Hemodynamics Using High-Frequency Micro-Ultrasound}

John Sun, Liis Lindvere, Martijn E. van Raaij, Adrienne Dorr, Bojana Stefanovic and F. Stuart Foster

Cold Spring Harb Protoc; doi: 10.1101/pdb.prot5495

\begin{tabular}{|c|c|}
\hline $\begin{array}{r}\text { Email Alerting } \\
\text { Service }\end{array}$ & Receive free email alerts when new articles cite this article - click here. \\
\hline \multirow{2}{*}{$\begin{array}{l}\text { Subject } \\
\text { Categories }\end{array}$} & Browse articles on similar topics from Cold Spring Harbor Protocols. \\
\hline & $\begin{array}{l}\text { Cell Biology, general (1382 articles) } \\
\text { Cell Imaging (525 articles) } \\
\text { Image Analysis (124 articles) } \\
\text { Imaging for Neuroscience (342 articles) } \\
\text { Imaging/Microscopy, general (579 articles) } \\
\text { In Vivo Imaging (334 articles) } \\
\text { In Vivo Imaging, general (168 articles) } \\
\text { Neuroscience, general (357 articles) } \\
\text { Visualization (524 articles) } \\
\text { Visualization, general (369 articles) }\end{array}$ \\
\hline
\end{tabular}

